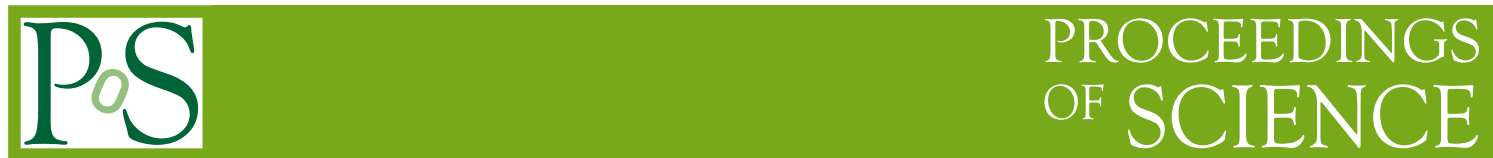

\title{
The emission distribution in SS433
}

\author{
Linda Schmidtobreick* \\ ESO, Chile \\ E-mail: 1schmidt@eso.org

\section{Katherine Blundell} \\ University of Oxford, UK \\ E-mail: katherine.blundell@physics.ox.ac.uk
}

\begin{abstract}
We present the results from a large observing campaign where we obtained 61 medium resolution spectra spread over three months. We thus cover roughly five orbits and about half a precession phase. In this paper we describe the analysis of the so-called "stationary" emission lines, which we use to compute Doppler-maps of the emission distribution in the SS433-system. The radial velocities of the individual line components have been analysed. Periodic variations with the orbital period are confirmed, but also variations on longer timescales are found. These long-term variation might be either either related to the precession phase or to some transient phenomen.
\end{abstract}

VI Microquasar Workshop: Microquasars and Beyond

September 18-22, 2006

Como, Italy

\footnotetext{
*Speaker.
} 


\section{Introduction}

SS433 has become famous as the first known relativistic jet source in the Galaxy. Red- and blue-shifted optical lines, indicating velocities of $0.26 c$, were discovered by Margon et al (1979a,b) and interpreted as being from gas accelerated in oppositely-directed jets (Fabian \& Rees [1979], Milgrom [1979]). Margon ([1984]) successfully fitted a kinematic precessing jet model, finding an intrinsic jet speed of $\approx 0.26 c$, a precession period of $\sim 163$ days, a cone opening angle of $\sim 20$, and an inclination to the line-of-sight of $\sim 80^{\circ}$. SS 433 is believed to be a binary system with an orbital period of 13.08 days which consists of a compact source accreting matter from a companion.

\section{Data and reduction}

The observations were performed between August and November 2004 using EMMI on ESO's New Technology Telescope (NTT) at La Silla, Chile. Grating \#6 together with a 0.5 arcsec slit was used to cover the wavelength range from 5800 to $8700 \AA$ with a spectral resolution of $2.2 \AA / F W H M$. Weather permitting, the individual exposure time was $300 \mathrm{~s}$, and usually one spectrum was observed per night. In total, 61 spectra were taken spread over three months and thus covering roughly five orbits and about half a precession phase.

The standard reduction of the data have been performed using IRAF. The spectra have been optimally extracted (Horne [1986]) and wavelength calibrated. No flux calibration was performed. Instead, the spectra have been normalised for the continuum using the program SPLOT kindly written and provided by Jochen Liske. The moving $\mathrm{H} \alpha$ jet lines were individually fitted for all spectra and subtracted to get a clean data set of stationary lines. All further analysis have been done using MIDAS.

\section{Results}

In Figure 1, the average spectrum of SS 433 is plotted. It has been constructed by averaging all 61 spectra, of which the moving jet lines were removed. The resulting $\mathrm{S} / \mathrm{N}$ of the average spectrum amounts to about 350 for the continuum.

The spectrum is dominated by the strong stationary $\mathrm{H} \alpha$ emission, but many other lines are present as well. In the red, the Paschen series from Paschen 11 up to the break is found. Other emission lines originate in low ionisation states of He I, O I, and C II. While the lines in the individual spectra show clear double-peak profiles, these are mainly washed-out in the average spectrum which only shows broad single-peak profiles. The average FWHM of the lines is around $15 \AA$, and slightly increasing with wavelength. However, $\mathrm{H} \alpha$ is much broader with $22 \AA$. With these values we derive the projected rotation velocity to roughly $520 \mathrm{~km} / \mathrm{s}$, while for $\mathrm{H} \alpha$, it is $1010 \mathrm{~km} / \mathrm{s}$.

The profiles of the emission lines change dramatically during our observing campaign (see Figure 2). While typical double-peak profiles of rotating discs are present at the beginning, at the later stages $\mathrm{H} \alpha$ is dominated by a strong broadening, and P Cygni absorption develops in most lines around $\mathrm{HJD}=2453294$. These are most likely connected to a radio burst, that happened around $\mathrm{HJD}=2453293$. The $\mathrm{H} \alpha$ broadening, instead, evolves smoothly over the whole time scale, and might thus be connected to the precession phase. 


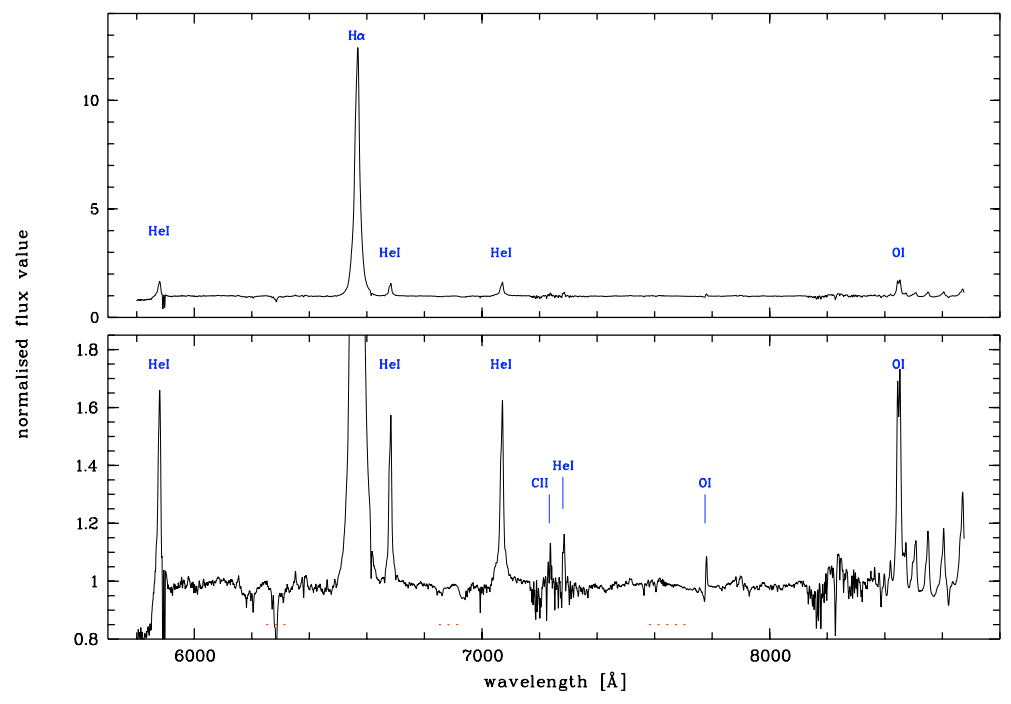

Figure 1: The average spectrum of SS433 in the range $580-870 \mathrm{~nm}$. The jet-lines have been removed before combining the individual spectra.
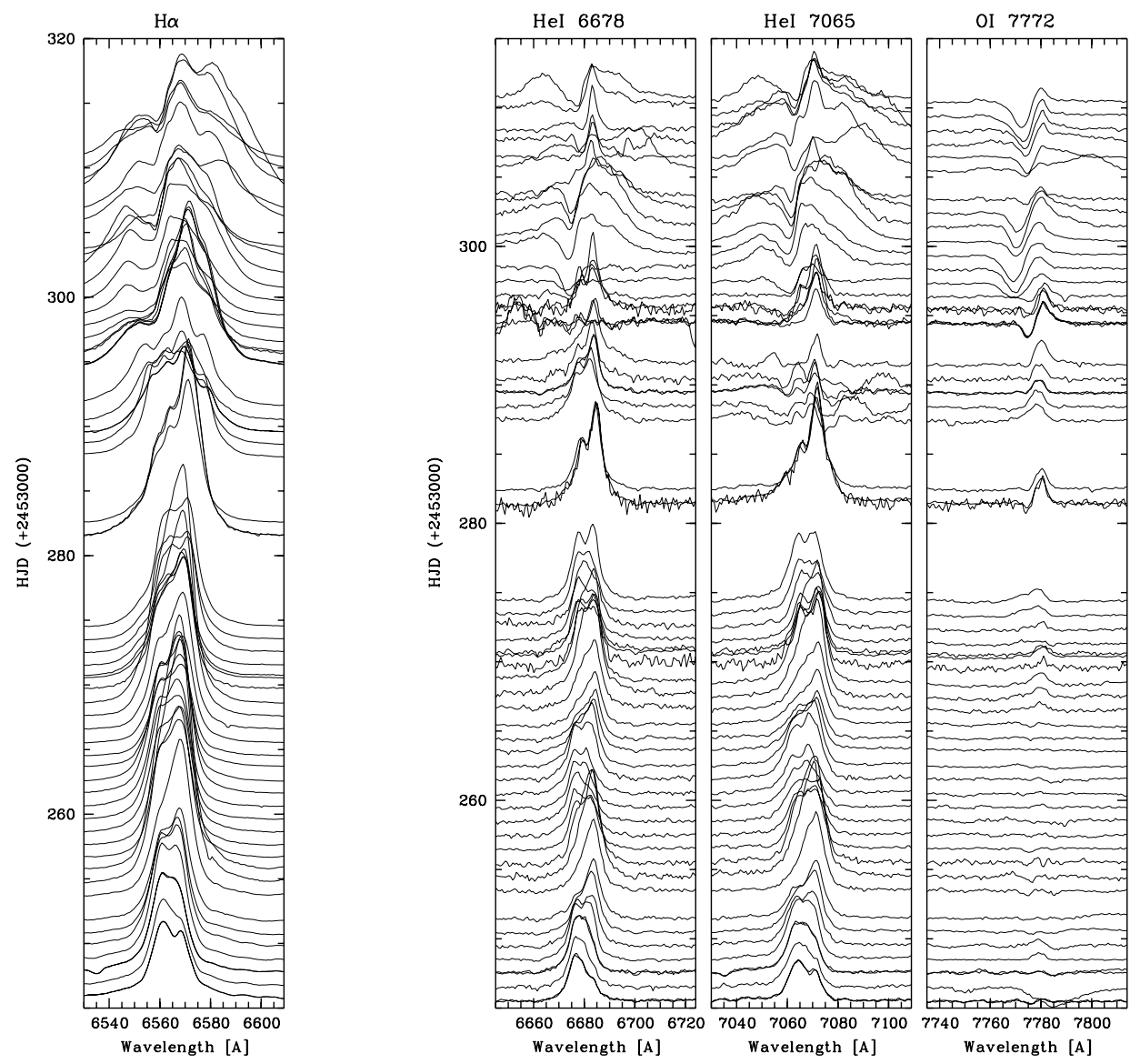

Figure 2: The evolution of the line profiles is demonstrated in 4 examples. The spectra of $\mathrm{He} \mathrm{I}$ and O I are enhanced by a factor of 7 with respect to $\mathrm{H} \alpha$. 

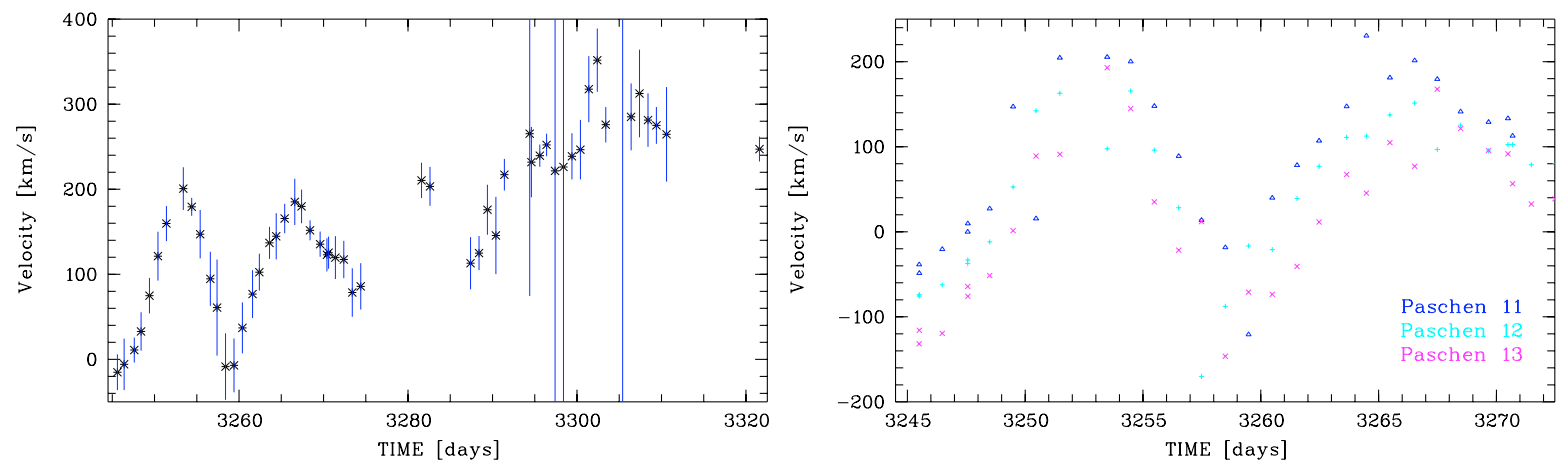

Figure 3: The left graph shows the averaged radial velocities of the measured lines (see text for details) plotted against time. On the right, the radial velocities of Paschen 11, 12, and 13 are plotted against time. The individual curves are shifted due to different apparent system velocities.
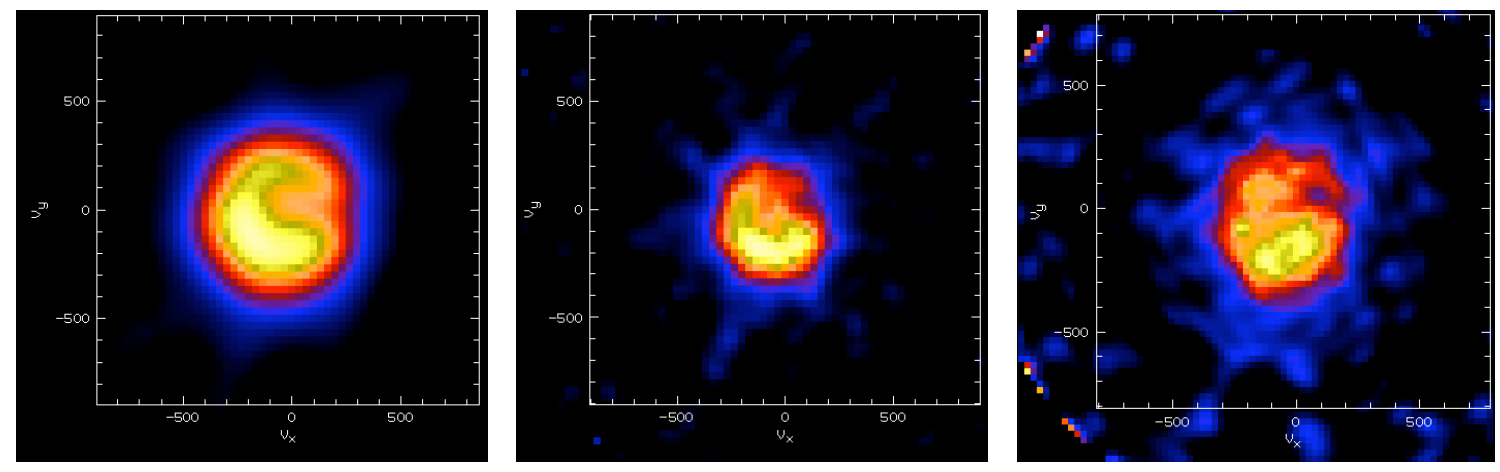

Figure 4: Doppler tomography using the $\mathrm{H} \alpha$ (left), He I (centre), and Paschen 11 (right) emission lines of the first 29 spectra. The orientation for all maps is such that the donor star is situated up, the phase angle is increasing anti-clockwise.

The radial velocities have been determined by fitting broad Gaussians to the emission lines. We have used the emission lines $\mathrm{H} \alpha, \mathrm{He} \mathrm{I}$ at $6678 \AA$ and $7065 \AA$, and Paschen 11, 12 and 13 and averaged these velocities. The radial velocities show the expected variation with the orbital period. From the first two orbits of the average velocities, we derive a radial velocity amplitude $K_{1}=$ $110 \mathrm{~km} \mathrm{~s}^{-1}$ and a system velocity $\gamma=100 \mathrm{~km} \mathrm{~s}^{-1}$.

However, both these values seem to increase throughout our observing campaign. While the variation of $K_{1}$ seems to depend strongly on the line that is used for its determination, the system velocity apparently increases systematically for all analysed lines and reaches a value of $\gamma=350 \mathrm{~km} \mathrm{~s}^{-1}$ before reaching a plateau and even starts decreasing again. Again, it is not clear, if this variation of the system velocity is connected to the radio burst or to the precession phase.

Plotting just the velocities for the Paschen lines, one realises that there is an offset between the radial velocity curves of the three lines. This shift in the apparent system-velocity might be explained by gravitational redshifting, due to the fact that different lines might originate in regions of the accretion disc that have different distances to the central compact object. This gravitational redshifting might also be responsible for the general increase of the apparent system velocity. Assuming that, depending on precession phase, parts of the inner disc become visible, where the 
gravitational redshift is higher, the apparent system velocity will depend on the precession phase. If the radio burst actually blew away part of the disc, which would also explain the PCyg profiles, this effect might increase, as even more of the inner disc regions are revealed.

The Doppler maps $I\left(v_{x}, v_{y}\right)$ display the flux emitted by gas moving with the velocity $\left(v_{x}, v_{y}\right)$ and thus show the emission distribution in velocity coordinates with the centre of mass at $(0,0)$. We have used the code of Spruit ([1998]) with a MIDAS interface replacing the original IDL routines (Tappert et al. [2003]). We computed maps for different emission lines, using only the first 29 spectra, and individual apparent system velocities for each line. All maps show a kind of ring-like feature, which would indicate an accretion disc. However, this ring is not closed and in general the intensity is much higher on the left side. This might be explained by an extended hot-spot region, where the material of the secondary star hits the accretion disc. However, its elongated structure rather hints to accretion happening via a stream in addition to the disc, which would indicate that the compact object inhabits a strong magnetic field. Polarimetric observations will show whether this interpretation is correct.

A more detailed analysis and interpretation of these data will be presented in a forthcoming paper.

\section{References}

[1979] A.C. Fabian, M.J. Rees, MNRAS 187, 13

[1986] K. Horne, PASP 98, 609

[1979] B. Margon, H.C. Ford, J.I. Katz, et al., ApJ 230, L41

[1979b] B. Margon, S.A. Grandi, R.P.S. Stone, H.C. Ford, ApJ 233, L63

[1984] B. Margon, ARAA 22, 507

[1979] M. Milgrom M., A\&A 76, L3

[1998] H. Spruit, preprint, astro-ph/9806141

[2003] C. Tappert, R. Mennickent, J. Arenas, et al., A\&A 408, 651 [astro-ph/ 0308235 ] 